\title{
SÚPER-DIVERSIDAD Y COMERCIO LOCAL \\ Las huellas de la gentrificación y de la migración transnacional en el centro de Santiago \\ SÚPER-DIVERSIDAD Y COMERCIO LOCAL \\ Las huellas de la gentrificación y de la migración transnacional en el centro de Santiago
}

\author{
Catalán Hidalgo, René \\ Instituto de Estudios Urbanos y Territoriales, Pontificia Universidad Católica de Chile \\ rlcatala@uc.cl
}

\section{RESUMEN}

La presente investigación aborda las transformaciones materiales de los espacios comerciales del centro de Santiago, a partir del concepto de súper-diversidad. Hablar de súper-diversidad en una ciudad del Sur Global como Santiago, implica incluir los fenómenos de gentrificación y de migración transnacional, siendo ellos y su desarrollo en la ciudad, claves para comprender un proceso reciente como el de la súper-diversificación. Para lograr este fin, se creó el Indicador de Potencial de Gentrificación el que permitió identificar la zona específica de Santiago, que corresponde a un polígono del Barrio Lira, el cual se abordó en la segunda etapa. Posteriormente se empleó análisis visual con fotografías tomadas en el barrio e imágenes obtenidas desde Google Street View, contraste que permite analizar las transformaciones recientes de los espacios comerciales de la zona seleccionada, de una perspectiva de la súper-diversificación de la ciudad de Santiago.

Palabras clave: súper-diversidad, gentrificación, gentrificación comercial, migración Bloque temático: La ciudad Poscrisis. Transformaciones socio-urbanas y políticas: Gentrificación.

\begin{abstract}
This research addresses the material transformations of the commercial spaces of the center of Santiago, using the concept of super-diversity. Talking about super-diversity in a city of the Global South such as Santiago, involves including the phenomena of gentrification and transnational migration, being these concepts and their development in the city, keys to understanding a recent process such as super-diversification. To achieve this aim, the Gentrification Potential Indicator was developed, these allowed the identification of the specific area of Santiago to be addressed in the second stage, which corresponds to a polygon of the Lira Neighborhood. Subsequently, visual analysis was used with photographs taken in the neighborhood and images obtained from Google Street View, a contrast that allows the analysis of recent transformations of the commercial spaces of the selected area, from a perspective of the super-diversification of the city of Santiago.
\end{abstract}

Keywords: super-diversity, gentrification, commercial gentrification, migration.

Topic: La ciudad Poscrisis. Transformaciones socio-urbanas y políticas: Gentrificación. 


\section{Introducción}

\subsection{Súper-diversidad y los espacios comerciales}

Uno de los conceptos claves par la compresión de la diversidad es el de multiculturalismo, el cual grafica el pluralismo presente en la ciudades, principalmente del Norte Global, gatillado por las importantes olas migratorias vinculadas con la caída de los diferentes colonialismos, y los grandes procesos migratorios motivados por causas humanitarias o económicas (Yanasmayan, 2011). El concepto de "súper-diversidad" (Vertovec, 2007) emerge para complejizar esta forma de entender la diversidad.

El concepto de súper-diversidad considera la interacción de otras variables como son los diferentes status migratorios, las distintas experiencias con el mercado laboral, los elementos de género y generacionales, los patrones de distribución espacial, entre otros factores; conformándose distinciones que no sólo complejizan lo que se entiende por diversidad, yendo más allá de la mera diversidad étnica.

La emergencia de este concepto tiene como hito clave, la publicación por la Alcaldía de Londres de un análisis de los resultados del Censo de 2001 bajo el titulo "The World in One City" ("El mundo en una ciudad"). Este análisis establecía la presencia de 179 nacionalidades en la ciudad, situándola como la ciudad con mayor multiculturalidad del mundo (Freedland, 2005). Al replicar este análisis en Santiago, vemos que en 2017 vivían personas provenientes de al menos 67 países $^{1}$ (INE, 2017b). Aunque esta cifra representaría cerca de un tercio de la multiculturalidad registrada en Londres, considerar esta diversidad desde la súper-diversidad, permite interrogarse por la complejidad de un escenario como el de esta ciudad.

\subsection{Espacios comerciales como espacios para la súper-diversidad}

Los espacios comerciales emergen como lugares de encuentro entre la sociedad y la economía, en donde la diversidad se intersecta y donde personas con características muy dispares no sólo se encuentran, sino que además se coordinan (Hiebert, Rath, \& Vertovec, 2015). Las bajas barreras de entradas en términos económicos, sociales y culturales facilitan la participación de la población migrante que se ha vuelto vulnerable producto del propio movimiento migratorio, viendo dificultada su completa inclusión es las diferentes esferas sociales. Estas características de estos espacios permiten entenderlos como lugares en donde se desarrolla la pertenencia en contextos marcados por la diversidad (Hall, 2009, 2012, 2013). Desde esta perspectiva entonces son un contexto clave para el estudio de la súper-diversidad gracias a sus características particulares que le dan elementos de "universalidad" a este tipo de espacios alrededor del mundo.

Frente a estas características universales de los espacios comerciales, cabe preguntarse por las particularidades que emergen en el contexto de una ciudad como Santiago y que le otorgan características distintas a la súper-diversidad derivada de sus procesos migratorios recientes.

\subsection{Súper-diversidad en el Sur Global: gentrificación como punto de inflexión}

¿Cuál es la característica propia de la súper-diversidad en un contexto como el de la ciudad de Santiago, en el marco del Sur Global? La respuesta a esta pregunta introduce a la gentrificación como elementos clave dentro del análisis: "En los países más desarrollados estamos acostumbrados a pensar en que quienes llegan a la ciudad se asientan primero en los espacios deteriorados del centro. Pero el proceso conjunto de gentrificación y migración en masa [del Sur Global] ha cambiado profundamente la geografía de los asentamientos migrantes, el que es principalmente un fenómeno suburbano" (Hiebert et al., 2015: 14).

1 Las cifras del Censo 2017 sobre países de origen, agrupan por continente ${ }^{\text {a los países con menor cantidad de migrantes. }}$ 
A diferencia de lo que ocurrió en el Norte Global, en el Sur Global los procesos masivos de migración, como el que atraviesa Chile en la actualidad, ${ }^{2}$ se entremezclan con procesos de gentrificación del centro de la ciudad. Los migrantes de esta región no tienen asegurados espacios específicos del centro de la ciudad como espacio de asentamiento, debiendo disputar estos espacios con los grupos medios que vuelven a vivir al centro de la ciudad (Ibid., 2015; Wessendorf, 2014).

Frente a este proceso de transformación de la ciudad atravesado por la súper-diversidad, los estudios recientes sobre la gentrificación en Santiago de Chile (Janoschka, 2016, 2016; López-Morales, 2013, 2015; LópezMorales, Shin, \& Lees, 2016; Sabatini, Rasse, Cáceres, Robles, \& Trebilcock, 2017) no han abordado el factor migración para comprender el panorama completo de estos cambios urbanos, situándose mayormente desde la oposición entre dos grupos en conflicto, es decir, entre migrantes transnacionales y la población "autóctona" del barrio, o entre esta última y los gentrificadores.

Desde el estudio de la gentrificación comercial en tanto, la escasa investigación tampoco abordan el efecto conjunto de ambos procesos, limitándose a analizar la activación y renovación de los espacios comerciales (Colodro, 2017) o a replicar la lógica del desplazamiento de los espacios residenciales a los espacios comerciales (Rivera \& Orozco, 2014).

De esta forma entonces es que emerge la pregunta que guía esta investigación: ¿cómo se transforma el entorno construido en los espacios comerciales de los barrios con alto potencial de gentrificación y con alta migración transnacional en la ciudad de Santiago, en un contexto de súper-diversidad? Esta pregunta es la introducción al problema de la construcción de la pertenencia en estos lugares, el cual guía la investigación que actualmente realizo en el marco de mi tesis de magíster.

\section{Marco conceptual}

A continuación, se presentan los principales conceptos que se emplearán en este análisis. Primero, el concepto de súper-diversidad el cual busca complejizar la compresión que se tiene de la diversidad en el contexto urbano global. Segundo, se presenta el concepto de gentrificación y las características propias del mismo para el contexto chileno. Tercero, el de gentrificación comercial, como un aspecto distinto a la gentrificación residencial, y que vincula al desplazamiento propio de la gentrificación con el consumo y la cotidianidad. Por último, el concepto de pertenencia (belonging), como marco para comprender la construcción y/o transformación de estos espacios.

\subsection{Súper-diversidad y el desafío de comprender las transformaciones urbanas desde la complejidad}

Vertovec (2007), formula el concepto de súper-diversidad al constatar la necesidad de abordar la diversidad más allá del habitual contexto étnico desde el que se la aborda. Desde esta perspectiva, el estudio de la diversidad debiera considerar una serie de variables adicionales como son los status legales migratorios, por los derechos y restricciones que ellos generan; las estrategias diversas de inserción en el mercado laboral; así como otros elementos propios de la interacción cotidiana como son el género, las relaciones intergeneracionales, las diferencias a nivel de capital cultural, entre otros factores.

Desde una formulación más reciente, Wessendorf describe la súper-diversidad: "caracterizada no sólo por una multiplicidad de diferentes minorías étnicas y de migrantes, sino que también por la diferenciación en términos de la historia de la migración, los antecedentes educacionales, la religión, el status legal, largo de la residencia

2 De acuerdo con los datos aportados por el INE, el porcentaje de migrantes internacionales en Chile pasó del 0,8\% en 1992 al 1,3\% en 2002, Ilegando en 2017 al 4,4\%. Cifra que para la Región Metropolitana 'epresentó un 7\% (INE, 2018). 
y los antecedentes económicos, entre las minorías étnicas y los migrantes, así como de la población blanca británica" (Wessendorf, 2014: 1).

Lo interesante de esta formulación es que va más allá del contexto migrante, incluyendo a la población inglesa blanca dentro de la conceptualización, desenmarcando el concepto de los límites de la migración.

Por último, Hiebert et al. (2015), propone una geografía del concepto, estableciendo una diferencia entre el Norte y Sur Global. Si en el Norte Global primero se dio la migración y luego la gentrificación, y estos últimos desplazaron a los primeros; en el Sur Global estos procesos se dan en conjunto, con migrantes y gentrificadores disputándose el centro de la ciudad, lo que a su vez genera una suburbanización de la gentrificación.

\subsection{Descolonizando el concepto de Gentrificación}

El concepto original de gentrificación acuñado por Glass (2010) para la ciudad de Londres, expresaba el asentamiento de grupos medios hacia las áreas centrales deterioradas de la ciudad, lo que implicaba tanto renovación o rehabilitación de los barrios y sus viviendas, y el desplazamiento de la población. Con algunos matices, este modelo original se fue expandiendo y se utilizó para comprender procesos similares en el resto de Europa, Estados Unidos y en países del Sur Global, la gentrificación se había convertido en un fenómeno global (Smith, 2002).

Más recientemente, Clark (2005: 263), ha definido el fenómeno como "la reestructuración espacial del área urbana por inyección de capital fijo en el mercado inmobiliario y de infraestructura, orientada al reemplazo de usuarios de ingresos medios-bajos por usuarios de poder económico superior, en contexto de mercantilización del suelo" (Clark, 2005), definición base para esta investigación.

Sin embargo, la globalización de este fenómeno ha enfrentado a los investigadores a la necesidad paralela de cuestionarse este concepto. Lees $(2000,2012)$ es quien realiza los primeros llamados en contra de lo que denomina un "urbanismo imitativo", esto es el uso de conceptos globales sin atención a su especificidad geográfica. Para el caso latinoamericano, la misma autora en conjunto con López-Morales y Shin (2016) han analizado las características del fenómeno para el contexto local, lo cual les ha permitido afirmar que existe una geografía particular de la gentrificación en América Latina.

Para el caso chileno en particular, López-Morales (2013) plantea que la gentrificación estaría garantizada por un recambio a gran escala, con considerable altura y densidad, del stock edificado residencial; proceso que es dominado por agentes productores y no por residentes, lo cual marca una diferencia importante respecto al contexto europeo y norteamericano (Casgrain \& Janoschka, 2013).

\subsection{Gentrificación comercial como acceso a la transformación de la cotidianidad}

Las conceptualizaciones presentadas sin embargo, no abordan la especificidad cotidiana del fenómeno, es por eso que se propone el concepto de "gentrificación comercial" para analizar la transformación desde la perspectiva de los espacios comerciales del barrio, concepto que ha tenido un abordaje menor en los estudios urbanos (Rojo, 2016)

Marcuse (1985) en su texto clásico sobre el desplazamiento entrevé este proceso gentrificación comercial, haciendo referencia al desplazamiento por condiciones externas a la vivienda mismas y que se centra en el barrio y su encarecimiento, impactando en el consumo debido al alza de los precios en las tiendas del sector o la aparición de nuevas tiendas enfocadas en una clientela distinta a la que habitualmente viven en el barrio. 
Para comprender la gentrificación comercial es clave el concepto de "desplazamiento por desposesión del hábitat" de Janoschka (2016), quien lo propone como una expansión de la "desposesión por acumulación" propuesta por Harvey (2005). El desplazamiento por desposesión del hábitat opera a un nivel económico, donde se inserta Harvey; un nivel político, por la labor directa o indirecta del Estado; además de un nivel simbólico y uno psicológico; son estos dos últimos niveles los que marcan la diferencia.

Como proceso simbólico, el desplazamiento expresa la reconfiguración de los espacios urbanos mediante su homogeneización, el cual expresa una pugna por la hegemonía cultural de estos espacios. La consideración simbólica del desplazamiento va muy a la par de la consideración psicológica, ya que el cambio simbólico impacta a nivel individual en forma de alienación de los habitantes, quienes experimentan la pérdida del lugar (Janoschka, 2016). Son estas dimensiones del desplazamiento, simbólica y psicológica, las que permiten una mejor comprensión de la gentrificación comercial y de la gentrificación como un proceso totalizador que opera a diferentes niveles y que supera los límites de la residencia.

González y Waley (2013) distinguen tres características del proceso de gentrificación comercial:

- La privatización y comodificación de las zonas centrales de la ciudad,

- El desplazamiento de los consumidores habituales que, en el caso particular,

- La transformación de estas zonas en una experiencia de consumo, mediante la fetichización de la comida y la oferta de un espacio sanitizado y comodificado.

Hubbard (2017) en tanto, conceptualiza a este proceso como "blanqueamiento" y como una "limpieza de clase" de los espacios comerciales del barrio, transformación que no sólo es cultural, sino que también de clase

En síntesis, se puede ver cómo es que el desplazamiento producido por la gentrificación comercial se vuelve crítico, si es que entendemos que la compra y el consumo en general, son la fuente principal de ciudadanía y de expresión creativa de la sociedad contemporánea (Zukin, 2008).

\subsection{El comercio migrante como espacio de pertenencia}

Los espacios comerciales, más allá de la transacción económica, son espacios de sociabilidad (Gonzalez, 2017; Mayor of London, 2017): "Las interacciones entre emprendedores y consumidores en los mercados callejeros son públicas. Los consumidores presencian transacciones y aprenden los hábitos/el habitus de el mercado en una especie de teatro público [...] La información sobre los precios es comunicada en público y los códigos de conducta son transmitidos de vendedor a vendedor y de consumidor a consumidor" (Hiebert et al., 2015).

Los espacios comerciales entonces, emergen como lugares en que significados y prácticas son transmitidos e incorporados (Zukin, 2005).

Esta capacidad de conexión no sólo refiere a significados y prácticas propias del contexto, los espacios comerciales además tienen la capacidad de conectar con espacios que están más allá, en lo que Doreen Massey ha denominado "sentido global de espacio" (citado en S. Hall, Finlay, \& King, 2018). Este carácter translocal es fundamental cuando analizamos los espacios comerciales ligados con la migración.

Al encontrarse en un contexto nacional distinto, las tiendas de migrantes transnacionales son espacios claves para la construcción de "pertenencia" en las comunidades de migrantes, ya que ellas posibilitan la sensación de "estar en casa" (Hall, 2009).

Un elemento adicional a la hora de analizar el comercio migrante es que éste no sólo se da en los marcos del comercio formal, acotado al espacio específico de una tienda, éste también se constituye en espacios informales dentro del espacio de la calle. Esto se produce gracias a las bajas barreras de ingreso que presentan 
los espacios comerciales. El comercio informal no sólo flexibiliza las barreras normativas, sino también las barreras idiomáticas, educacionales y de capacitación (Hiebert et al., 2015).

El comercio entonces, sea formal o informal, gracias a su capacidad de comprimir el tiempo y el espacio, permite el entrecruce de personas con orígenes y culturas distintas, en un mismo lugar, siendo la forma principal mediante la cual lo global puede insertarse en lo local (Hiebert et al., 2015).

\section{MARCO METODOLÓGICO}

Dado que el objetivo transversal es el estudiar la transformación de espacios comerciales a nivel material, se empleó una metodología mixta para captar los diferentes aspectos de las transformaciones urbanas (Hwang, 2015; Loukaitou-Sideris, Gonzalez, \& Ong, 2017; Mayor of London, 2017).

\subsection{Identificación de zonas: Construcción del Indicador de Potencial de Gentrificación}

Al ser la súper-diversificación un concepto complejo y multivariable, la definición de la zona en donde se realizó el análisis posterior se hizo de forma deductiva, mediante la identificación espacial de los dos fenómenos que la caracterizan: gentrificación y migración. Para ello se construyó el Indicador de Potencial de Gentrificación (IPG) a nivel de la Región Metropolitana de Santiago, el que luego fue contrastado con el porcentaje de migración presente en las zonas identificadas mediante el IPG, el cual debía ser significativamente mayor al 7\% regional (INE, 2017b). De este modo, ambos parámetros permitieron realizar la selección.

Respecto a la temporalidad de este análisis, se consideraron 5 años en base a la pregunta censal "¿En qué comuna o país vivía en abril de 2012?" (INE, 2017a). ${ }^{3}$

Cabe señalar, que el IPG busca expresar la transformación en los diferentes territorios del Gran Santiago, por ende, el cálculo considera la variación porcentual dentro del período seleccionado, por lo que un número más alto en cualquiera de las dimensiones, indica una mayor variación entre ambos períodos y no una magnitud mayor del fenómeno medido.

La operacionalización del IPG entonces, queda expresada en la Tabla №1 la que muestra sus seis dimensiones, además de los indicadores utilizados y sus fuentes respectivas.

\footnotetext{
${ }^{3}$ Este período se aumento a 15 años para el caso de la información censal, 2002-2017, debido a las limitaciones del Censo 2012 (Contraloría General de la República, 2014).
} 


\begin{tabular}{|c|c|c|c|c|}
\hline Índice & Subíndice & Variable(s) & Período & Fuente \\
\hline \multicolumn{2}{|l|}{ Rejuvenecimiento } & Edad jefa(e) de hogar & $2002-2017$ & $\begin{array}{l}\text { Censo de Población y } \\
\text { Vivienda } 2017 \text { Censo Nacional } \\
\text { de Población y de Vivienda }\end{array}$ \\
\hline \multirow[t]{7}{*}{$\begin{array}{l}\text { Índice Socio } \\
\text { Material Territorial }\end{array}$} & \multirow[t]{2}{*}{$\begin{array}{l}\text { Aumento de la } \\
\text { escolaridad }\end{array}$} & $\begin{array}{l}\text { Curso o año más alto } \\
\text { aprobado }\end{array}$ & & 2002 \\
\hline & & $\begin{array}{l}\text { Nivel del curso más } \\
\text { alto aprobado }\end{array}$ & & \\
\hline & \multirow{3}{*}{$\begin{array}{l}\text { Mejora en el } \\
\text { Índice de } \\
\text { Materialidad }\end{array}$} & $\begin{array}{l}\text { Material de los muros } \\
\text { exteriores }\end{array}$ & & \\
\hline & & $\begin{array}{l}\text { Material en la cubierta } \\
\text { del techo }\end{array}$ & & \\
\hline & & $\begin{array}{l}\text { Material de } \\
\text { construcción del piso }\end{array}$ & & \\
\hline & \multirow{2}{*}{$\begin{array}{l}\text { Disminución del } \\
\text { hacinamiento }\end{array}$} & Cantidad de personas & & \\
\hline & & $\begin{array}{l}\text { Número de pieza } \\
\text { usadas } \\
\text { exclusivamente como } \\
\text { dormitorio }\end{array}$ & & \\
\hline \multicolumn{2}{|l|}{ Densificación } & Cantidad de personas & & \\
\hline \multicolumn{2}{|c|}{ Aumento Valor del Suelo } & Valor del suelo & 2012-2017 & $\begin{array}{l}\text { Observatorio de Ciudades UC, } \\
\text { en base a información del } \\
\text { Servicio de Impuestos Internos }\end{array}$ \\
\hline \multirow{2}{*}{\multicolumn{2}{|c|}{$\begin{array}{l}\text { Altura máxima en las edificaciones } \\
\text { Edificaciones de uso mixto }\end{array}$}} & Número de pisos & 2012-2017 & $\begin{array}{l}\text { Permisos de Edificación, } \\
\text { Instituto Nacional de }\end{array}$ \\
\hline & & Destino & 2012-2017 & Estadísticas \\
\hline
\end{tabular}

Tabla 1: Operacionalización Indicador de Potencial de Gentrificación. Fuente: elaboración propia.

A continuación, se explica cada una de las dimensiones de este indicador:

- Rejuvenecimiento: en base a la edad de la jefa o el jefe de hogar, indica cómo la edad promedio de cada zona disminuye.

- I Índice Socio Material Territorial (ISMT): este índice es construido en base a la metodología utilizada por el Observatorio de Ciudades UC (Observatorio de Ciudad UC, s. f.), y permite condensar la mejora en las condiciones de habitabilidad de los hogares y el nivel socioeconómico de los mismos, empleándose tres subíndices construidos en base a siete variables censales.

o Índice de escolaridad: la escolaridad se mide en años de escolaridad, mediante la combinación entre el último año o curso aprobado y el nivel educacional correspondiente.

o Índice de materialidad: este índice se compone de tres variables que recogen la materialidad de la vivienda a partir del piso, el techo y las paredes, en base a los criterios utilizados por la Encuesta de Caracterización Socioeconómica (Casen) (Observatorio Social, 2017).

o Índice de hacinamiento: este índice se construye a partir de la cantidad de piezas con uso exclusivo para dormitorio disponibles en la vivienda respecto al total de personas que viven en el hogar (Ibid., 2017).

- Densificación: esta se calcula a partir del total de personas que viven por hectáreas. 
- Aumento del valor del suelo: el valor del suelo se obtuvo a partir de la información dispuesta por el Servicio de Impuestos Internos y catalogada por el OCUC.4

- Altura máxima en las edificaciones: considerar la altura máxima de los permisos de edificación otorgados permite captar la reglamentación existente en materia de instrumentos de planificación territorial, ya que los permisos se otorgan en base a la normativa.

- Edificaciones de uso mixto: también desde la perspectiva de la planificación territorial, el destino de uso de las edificaciones autorizadas, permiten captar dicha normativa.

Adicionalmente, como discriminante base, se establecen dos condicionantes mandatorias: el aumento en la escolaridad y el aumento en la densificación. El aumento en la escolaridad se selecciona por el ser el mejor proxy del ingreso percibido de acuerdo con la Función de Mincer (1974), lo que permite dar cuenta del recambio de habitantes de perfil socioeconómico medio-bajo por un perfil superior. El aumento en la densidad en tanto, se selecciona por ser la cualidad clave que identifica López-Morales (2013) como característica propia de la gentrificación en el contexto de Santiago de Chile. De este modo, las zonas que no cumplían con estos requisitos obtuvieron un puntaje "0" en el IPG.

\subsection{Identificación de zonas: Análisis espacial}

En esta etapa se utilizó el análisis estadístico espacial denominado "Indicador Local de Asociación Espacial" (LISA, por sus siglas en inglés) (Anselin, 2010), el cual permite reconocer el agrupamiento de unidades territoriales en base a una variable específica (Andresen, 2011; Moons, Brijs, \& Wets, 2008).

Cada uno de los seis indicadores sirvieron de suministro para realizar un análisis LISA parcial y la superposición de cada uno de estos análisis permitió calcular un índice sumatorio con valores que van entre el 0 y el 6 , en base a los valores High-High. Es decir que, a mayor valor del IPG existe un mayor potencial de gentrificación de la zona.

\subsection{Cambio en los aspectos materiales y simbólicos de los espacios comerciales: Análisis visual y entrevistas}

Los cambios en los aspectos materiales y simbólicos de la zona seleccionada fueron abordados mediante un análisis visual. Tras la selección de la zona se fotografiaron las fachadas de los todos los espacios comerciales -comercio establecido- de la zona seleccionada. Posteriormente, estas imágenes fueron contrastadas con el registro histórico dispuesto en la plataforma Google Street View (GSV) de Google, análisis comparativo que toma como referencia el trabajo de Hwang (2015).

El uso de este análisis permitió que las características identificadas a partir del registro in situ fuesen contrastadas e identificar las transformaciones de los espacios comerciales de la zona seleccionada, expresada en la materialidad de sus espacios comerciales.

\section{Resultados}

\subsection{La zona seleccionada}

El análisis espacial arrojó como resultado un cuadrante perteneciente al Barrio Lira de la Comuna de Santiago, que se delimita por las calles Argomedo (norte), 10 de julio (sur), Vicuña Mackenna (oriente) y Portugal 
(poniente), el cual contaba con el mayor potencial de gentrificación, además de contar con un porcentaje de migración del 22\%, el Mapa №1 y la Tabla №2 muestran las principales características de la zona seleccionada.

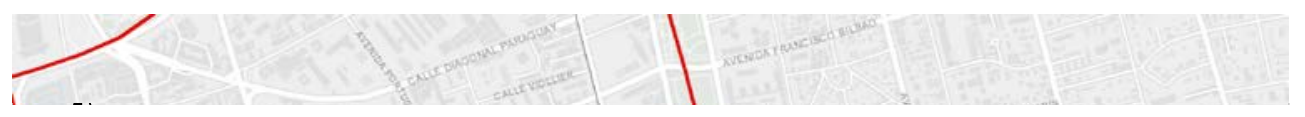

Henriquez

f. Supermercado Líder Express

g. Supermercado Tottus

Mapa 1: Potencial de gentrificación (índice) y principales características de la zona. Fuente: Elaboración propia.

\begin{tabular}{|l|l|}
\hline Zona & Indicador \\
\hline Promedio de personas en el hogar & 1,8 personas \\
Vulor del suelo & $34 \mathrm{UF} / \mathrm{m}^{2}$ \\
Densidad & 8,5 \\
$\%$ de jefatura femenina & 342,8 háb./há. \\
Edad promedio jefe(a) de hogar & $44 \%$ \\
Escolaridad promedio & 36,6 años \\
En esta comuna & 16,3 años \\
Otra comuna & $37 \%$ \\
Otro país & $43 \%$ \\
En esta comuna & $19 \%$ \\
Otra comuna & $9 \%$ \\
Otro país & $67 \%$ \\
Otros países de Sudamérica & $22 \%$ \\
\hline
\end{tabular}

Tabla 2: Tabla descriptiva de las zonas seleccionadas para el estudio. Fuente: Elaboración propia en base a datos INE y SII. 
Según vemos en la tabla №2 Santiago oriente tiene una superficie de 8,5 hectáreas y una densidad 342,8 hab./há. El valor del metro cuadrado es de 34 UF/m2 y en los hogares están compuestos en promedio por 1,8 personas.

A nivel socioeconómico, la zona tiene una jefatura femenina del $44 \%$, la edad promedio del jefe(a) de hogar es de 36,6 años, quien en promedio tiene 16,3 años de escolaridad.

En materia migratoria, sólo un 37\% de sus hogares vivía en la misma comuna hace 5 años, mientras el 22\% nació en otro país. De este $22 \%$ de migrantes, el origen principal es Venezuela con un $10 \%$ de la población de la zona, le siguen Colombia con un $4 \%$, Perú y los países europeos, ambos con un $2 \%$.

El mapa №2 también muestra que esta zona se encuentra próxima a dos líneas -Línea 3 y Línea 5- y dos estaciones del Metro -Irarrázaval y Santa Isabel-. Además, cuenta con dos supermercados, dos ferias libres. Adicionalmente, la zona se encuentra en las cercanías del Barrio Italia y el Parque Bustamante.

\subsection{Los cambios materiales del barrio}

A través del contraste de registros fotográficos se pudo distinguir cuatro tipos de intervenciones o estrategias de transformación comercial del barrio.

Comercio de menor escala: se trata del tipo de comercio que más se ha multiplicado en el barrio. Sus principales estrategias son el aumento de la variedad de su oferta, principalmente la de productos vinculados con la migración latinoamericana que se ha asentado en el barrio: harina para arepas, distintos tipos de empanadas, chicha venezolana, plátanos verdes, entre otros.

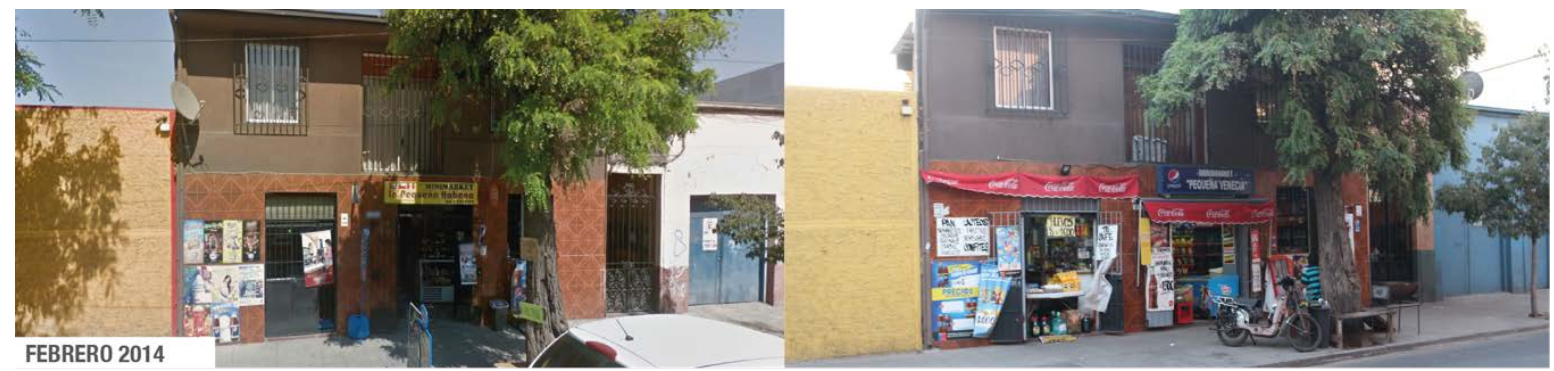

Fotografía 1: Minimarket, Fray Camilo Henríquez. ${ }^{5}$ Fuente: Elaboración propia y registro obtenido desde GSV.

Comercio migrante: la escala de este comercio también es pequeña y el cambio pasa principalmente por el tipo de productos ofrecidos, cuya variedad viene dada por el ingreso de productos o servicios que no sólo responden a la demanda de las comunidades de migrantes que se asientan en el barrio, ellos también vienen a sincretizarse con la demanda nacional. A diferencia de lo que ocurre con los minimarkets, donde muchos de los dueños son autóctonos, pero quienes atienden son migrantes, en las peluquerías y barberías, es donde se presencia el mayor ingreso de lo que podrías denominar al migrante "emprendedor".

${ }^{5}$ En todas las imágenes comparativas presentadas, la fotografía de la derecha fue tomada en enero 2019. 


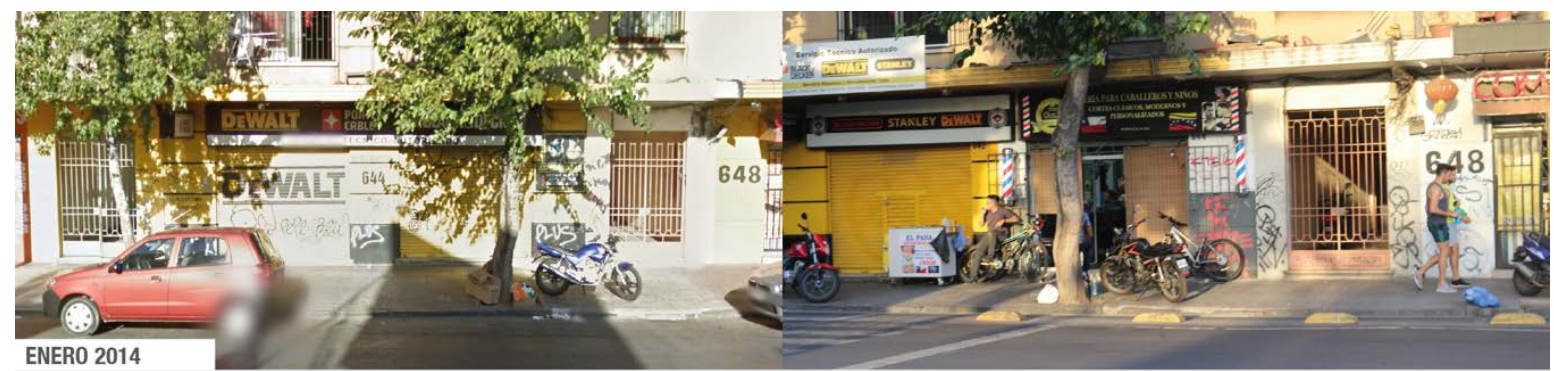

Fotografía 2: Barbería venezolana, Portugal. Fuente: Elaboración propia y registro obtenido desde GSV.

Supermercados de barrios: si bien es el de mayor escala y, por ende, el más intrusivo para el contexto barrial, este tipo de comercio mantiene códigos conocidos y aunque representa una amenaza para la forma en que tradicionalmente se concibe la relación cliente-locatario en un barrio, este formato colabora en la economía doméstica, debido a sus menores precios relativos:

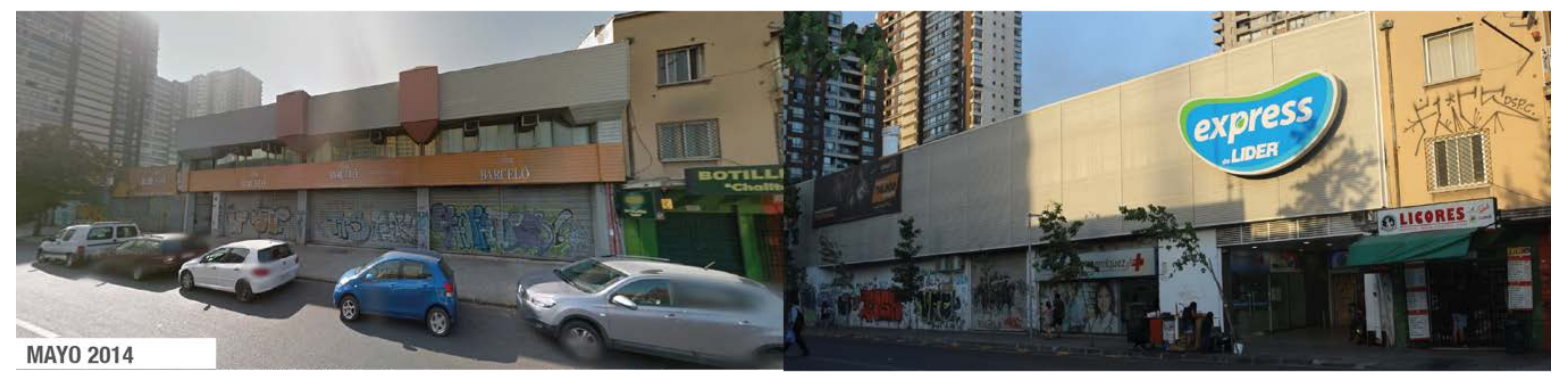

Fotografía 3: Supermercado, Avenida Portugal. Fuente: Elaboración propia y registro obtenido desde GSV.

Sofisticación del comercio: este tipo de comercio tiende a mantener una escala reducida y es, probablemente, el último en llegar, y se caracteriza por el cambio tanto en la oferta como en los aspectos estéticos, puntos claves que diferencian a este tipo de comercio y lo alejan del comercio de barrio habitual. En esta categoría también se encuentra la oferta gastronómica renovada y con un toque gourmet.

Este tipo de comercio además introduce claros patrones de embellecimiento, que se traduce en la recuperación de espacios arquitectónicos (fotografía $\mathrm{N}^{0} 6$ ) o el uso de coloridos murales comisionados que renuevan las fachadas de tiendas (fotografía Nº4).

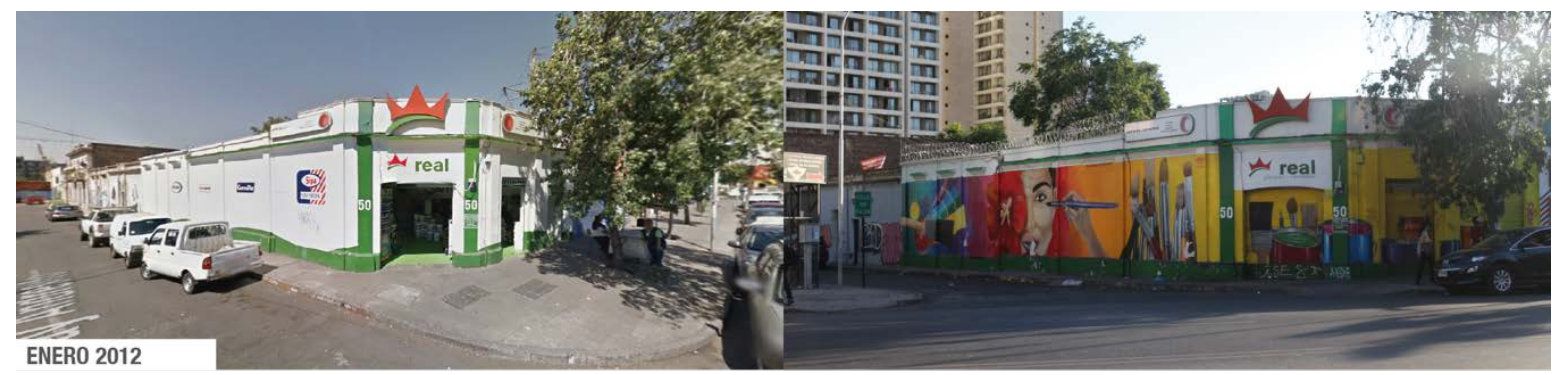

Fotografía 4: Mural en pinturería, Avenida 10 de julio. Fuente: Elaboración propia y registro obtenido desde GSV. 


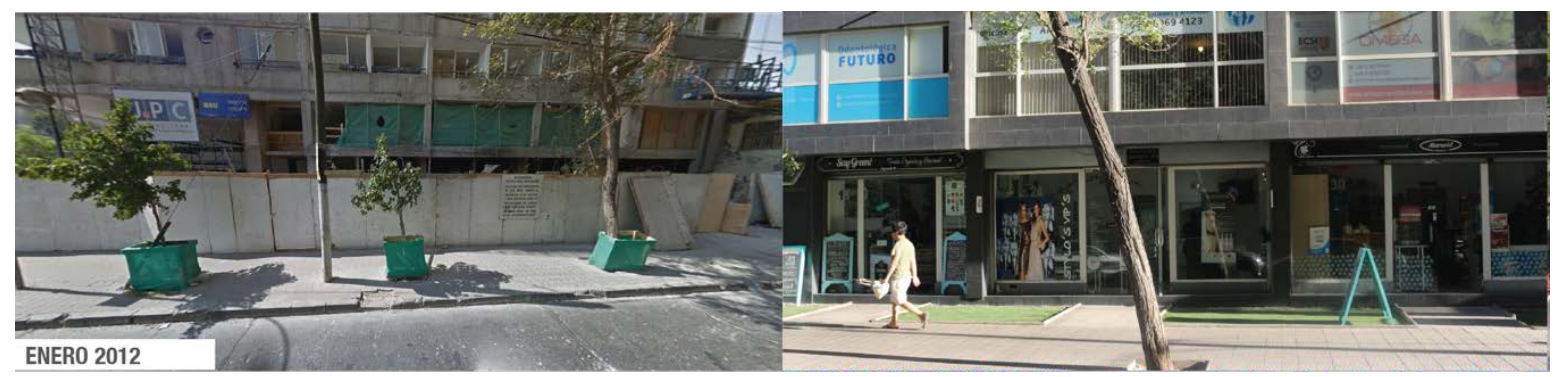

Fotografía 5: Placa comercial, Argomedo. Fuente: Elaboración propia y registro obtenido desde GSV.

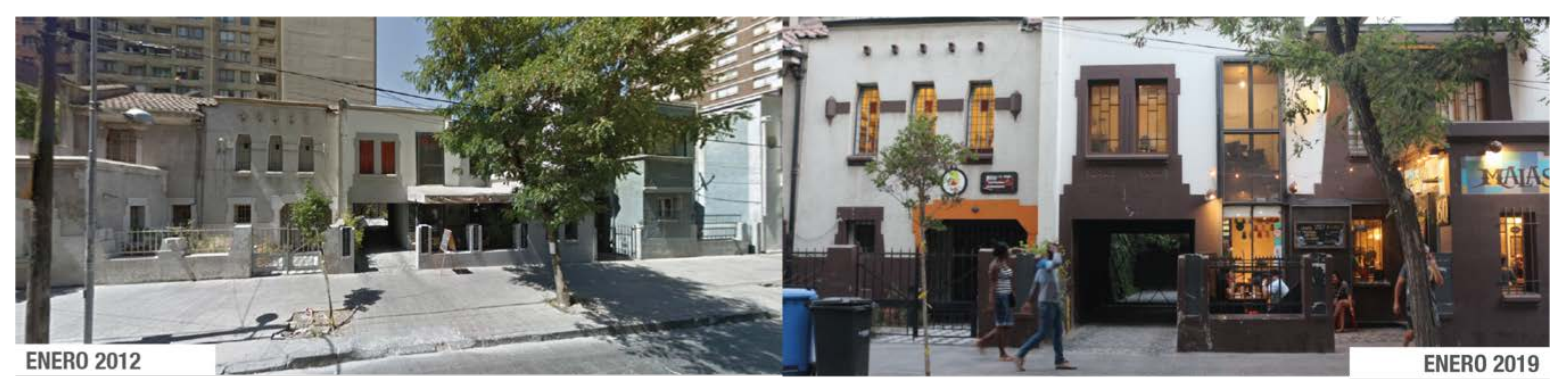

Fotografía N6: Bar Restaurante, Argomedo. Fuente: Elaboración propia y registro obtenido desde GSV.

\section{Conclusiones}

La presente investigación buscaba introducirse en la investigación de la transformación barrial de la mano del comercio, indagando en procesos de súper-diversificación, gentrificación comercial y migración, como un desplazamiento que opera cambiando los espacios comerciales, proceso que está en la base del desplazamiento por desposesión del hábitat. Este proceso queda inscrito en el espacio construido y constituye la base para entender procesos de cambio simbólico y en las prácticas en los espacios comerciales.

Es difícil pronosticar si en el Barrio Lira se producirá un fenómeno de consolidación de la gentrificación comercial, del comercio migrante o un híbrido súper-diverso de ambos. Se ofrecen al análisis desde los estudios urbanos sin embargo espacios que responden a nuevas lógicas de transformación y que permiten complejizar la comprensión de la transformación urbana en el contexto de Santiago de Chile.

Adicionalmente ayuda a comprender el proceso de gentrificación desde un análisis que involucre la complejidad de los procesos urbanos, expandiendo su significado más allá de los procesos experimentados por espacios como el Barrio Italia, o de entender a la gentrificación ligada simplemente a la pérdida de la vivienda, sino también como la pérdida del hábitat, entendiendo al barrio desde el acceso al consumo que provee.

Resta en la etapa siguiente y abordar el fenómeno desde aspectos simbólicos y desde las prácticas, y cómo ella se transforman.

\section{BIBLIOGRAFÍA}

ANDRESEN, M. A. (2011). Estimating the probability of local crime clusters: The impact of immediate spatial neighbors. Journal of Criminal Justice, 39(5), 394-404. https://doi.org/10.1016/j.jcrimjus.2011.05.005

ANSELIN, L. (2010). Local Indicators of Spatial Association-LISA. Geographical Analysis, 27(2), 93-115. https://doi.org/10.1111/j.1538-4632.1995.tb00338.x 
CASGRAIN, A., \& JANOSCHKA, M. (2013). Gentrificación y resistencia en las ciudades latinoamericanas EI ejemplo de Santiago de Chile. Andamios, Revista de Investigación Social, 10(22), 19. https://doi.org/10.29092/uacm.v10i22.265

CLARK, E. (2005). The Order and Simplicity of Gentrification A Political Challenge. En Gentrification in a Global Context (pp. 261-269). London; New York: Routledge, Taylor \& Francis Group.

COLODRO, U. (2017). La gentrificación comercial, ¿un proceso de activación del espacio público urbano?: Los casos de Santiago (Chile) y Seúl (Corea del Sur). Entretextos, 9(26), 47-57.

GLASS, R. (2010). Aspects of Change. En The Gentrification Reader (p. 1). London; New York: Routledge.

GONZALEZ, S. (2017). Contested markets, contested cities: Gentrification and urban justice in retail spaces (1 Edition). New York: Routledge.

GONZALEZ, S., \& WALEY, P. (2013). Traditional Retail Markets: The New Gentrification Frontier? Antipode, 45(4), 965-983. https://doi.org/10.1111/j.1467-8330.2012.01040.x

HALL, S. (2009). Being at Home: Space for Belonging in a London Caff. Open House International, 34(3), 8187 - (2012). City, street and citizen: The measure of the ordinary. London: Routledge - (2013). The politics of belonging. Identities, 20(1), 46-53. https://doi.org/10.1080/1070289X.2012.752371

HALL, S., FINLAY, R., \& KING, J. (2018). The Migrant Street. En The SAGE Handbook of the 21st Century City (pp. 464-477). SAGE Inc.

HARVEY, D. (2005). The new imperialism (1. issued as paperback). Oxford: Oxford Univ. Press.

HIEBERT, D., RATH, J., \& VERTOVEC, S. (2015). Urban markets and diversity: Towards a research agenda. Ethnic and Racial Studies, 38(1), 5-21. https://doi.org/10.1080/01419870.2014.953969

HUBBARD, P. (2017). The Battle for the High Street. https://doi.org/10.1057/978-1-137-52153-8

HWANG, J. (2015). Gentrification in Changing Cities: Immigration, New Diversity, and Racial Inequality in Neighborhood Renewal. The ANNALS of the American Academy of Political and Social Science, 660(1), 319340. https://doi.org/10.1177/0002716215579823

JANOSCHKA, MI. (2016). Gentrificación, desplazamiento, desposesión: Procesos urbanos claves en América Latina. Revista INVI, 31(88), 27-71.

LEES, L. (2000). A reappraisal of gentrification: Towards a 'geography of gentrification'. Progress in Human Geography, 24(3), 389-408. https://doi.org/10.1191/030913200701540483 - (2012). The geography of gentrification: Thinking through comparative urbanism. Progress in Human Geography, 36(2), 155-171. https://doi.org/10.1177/0309132511412998

LÓPEZ-MORALES, E. (2013). Gentrificación en Chile: Aportes conceptuales y evidencias para una discusión necesaria. Revista de Geografía Norte Grande, (56), 31-52. https://doi.org/10.4067/S071834022013000300003 - LÓPEZ-MORALES, E. (2015). Gentrification in the global South. City, 19(4), 564-573. https://doi.org/10.1080/13604813.2015.1051746

LÓPEZ-MORALES, E., SHIN, H. B., \& LEES, L. (2016). Latin American gentrifications. Urban Geography, 37(8), 1091-1108. https://doi.org/10.1080/02723638.2016.1200335

LOUKAITOU-SIDERIS, A., GONZALEZ, S., \& ONG, P. (2017). Triangulating Neighborhood Knowledge to Understand Neighborhood Change: Methods to Study Gentrification. Journal of Planning Education and Research, 0739456X1773089. https://doi.org/10.1177/0739456X17730890

MARCUSE, P. (1985). Gentrification, Abandonment, and Displacement: Connections, Causes, and Policy Responses in New York City. Urban Law Annual; Journal of Urban and Contemporary Law, 28(1), 195-240. 
MAYOR OF LONDON. (2017). High Streets for all. London.

MCFARLANE, C. (2011). Assemblage and critical urbanism. City, 15(2), 204-224. https://doi.org/10.1080/13604813.2011.568715

MINCER, J. (1974). Schooling, experience, and earnings. New York: National Bureau of Economic Research; distributed by Columbia University Press.

MINTCHEV, N., \& MOORE, H. L. (2018). Super-diversity and the prosperous society. European Journal of Social Theory, 21(1), 117-134. https://doi.org/10.1177/1368431016678629

MOONS, E., BRIJS, T., \& WETS, G. (2008). Hot Spot Analysis: Improving a Local Indicator of Spatial Association for Application in Traffic Safety. En Computational Science and Its Applications ICCSA 2008 (pp. 221-231). Verlag Berlin Heidelberg: Springer.

OBSERVATORIO DE CIUDADES UC. (s. f.). Metodología OCUC ISMT. sin publicar.

ROJO, F. (2016). La gentrificación en los estudios urbanos: Una exploración sobre la producción académica de las ciudades. Cadernos Metrópole, 18(37), 697-719. https://doi.org/10.1590/2236-9996.2016-3704

SABATINI, F., RASSE, A., CÁCERES, G., ROBLES, M. S., \& TREBILCOCK, M. P. (2017). Promotores inmobiliarios, gentrificación y segregación residencial en Santiago de Chile. Revista Mexicana de Sociología, (2), 229-260.

SMITH, N. (2002). New Globalism, New Urbanism: Gentrification as Global Urban Strategy. Antipode, 34(3), 427-450. https://doi.org/10.1111/1467-8330.00249

VERTOVEC, S. (2007). Super-diversity and its implications. Ethnic and Racial Studies, 30(6), 1024-1054. https://doi.org/10.1080/01419870701599465

WESSENDORF, S. (2014). Commonplace Diversity: Social Relations in a Super-Diverse Context. https://doi.org/10.1057/9781137033314

YANASMAYAN, Z. (2011). Concepts of Multiculturalism and Assimilation. En Interculturalism. Europe and its Muslims in Search of Sound Societal Models (pp. 17-27). Brussels: Centre for European Policy Studies.

ZUKIN, S. (2005). Point of purchase: How shopping changed American culture (1st paperback ed). New York: Routledge - (2008). Consuming Authenticity: From Outposts of Difference to Means of Exclusion. Cultural Studies, 22(5), 724-748. https://doi.org/10.1080/09502380802245985

\section{Fuentes electrónicas}

CONTRALORÍA GENERAL DE LA REPÚBLICA. (2014). Informe Investigación Especial (No 3). Recuperado de Contraloría General de la República website: http://transparencia.ine.cl/auditorias/informe_3.pdf

FREEDLAND, J. (2005, julio 15). The world in one city. The Guardian. Recuperado de https://www.theguardian.com/uk/2005/jul/15/july7.uksecurity9

INE, I. N. DE E. (2017a). Cuestionario Censo 2017. Recuperado de https://redatamine.ine.cl/cuestionarios/CVP2017.pdf - (2017b). WEB DISEMINACIÓN CENSO 2017. Recuperado 18 de abril de 2019, de https://resultados.censo2017.cl/Region?R=R13 - (2018). Características sociodemográficas de la inmigración internacional en Chile [Síntesis de Resultados]. Recuperado de https://www.ine.cl/docs/defaultsource/demogr\%c3\%a1ficas-y-vitales/inmigraci\%c3\%b3n/s\%c3\%adntesis.pdf?sfvrsn=795659d2 4

OBSERVATORIO SOCIAL. (2017). Archivo histórico de Encuesta Casenâ $\square$ "Vivienda. Recuperado 26 de junio de 2019, de http://observatorio.ministeriodesarrollosocial.gob.cl/casen/casen_def_vivienda.php 
RIVERA, M., \& OROZCO, H. (2014). Gentrificación comercial en Santiago Centro. Recuperado 19 de abril de 2019, de Scribd website: https://es.scribd.com/document/243897958/GENTRIFICACION-COMERCIAL-ENSANTIAGO-CENTRO-Maite-Rivera-y-Hernan-Orozco-pdf 